\title{
Diet of invasive lionfish (Pterois volitans and P. miles) in Bermuda
}

\author{
Corey Eddy ${ }^{1, *}$, Joanna Pitt ${ }^{2}$, James A. Morris Jr. ${ }^{3}$, Struan Smith ${ }^{4}$, \\ Gretchen Goodbody-Gringley ${ }^{5}$, Diego Bernal ${ }^{1}$ \\ ${ }^{1}$ University of Massachusetts Dartmouth, 285 Old Westport Road, Dartmouth, MA 02747, USA \\ ${ }^{2}$ Department of Environment and Natural Resources, Government of Bermuda, PO Box CR52, Crawl CRBX, Bermuda \\ ${ }^{3}$ National Oceanic and Atmospheric Administration, 101 Pivers Island Road, Beaufort, NC 28516, USA \\ ${ }^{4}$ Bermuda Natural History Museum/Bermuda Aquarium Museum and Zoo, PO Box FL145, Flatts FL BX, Bermuda \\ ${ }^{5}$ Bermuda Institute of Ocean Sciences, 17 Biological Lane, Ferry Reach, St George's, Bermuda
}

\begin{abstract}
As a generalist and opportunistic predator, lionfish (Pterois volitans and P. miles) consume large quantities of juvenile reef fish and invertebrates, as well as the adults of small-bodied species. To better understand the impacts of these fishes upon invaded coral reef ecosystems, we describe the feeding habits of invasive lionfish in Bermuda based on stomach contents analysis, and the influence that environmental factors have on their diet via spatial and temporal changes in prey availability. Relative to other regions throughout the northwestern Atlantic, lionfish in Bermuda consume a greater proportion of crustaceans, and their diet appears to rely upon the relative abundance of available prey species. A poorly -known crustacean, the red night shrimp Cinetorhynchus rigens, is the species of greatest importance to the diet of Bermuda lionfish. Currently, herbivorous fishes do not make a major contribution to their diet, although the lionfish frequently target both ecologically (e.g. bluehead wrasse Thalassoma bifasciatum) and economically important species (e.g. Atlantic creolefish Paranthias furcifer).
\end{abstract}

KEY WORDS: Invasive lionfish $\cdot$ Feeding ecology $\cdot$ Stomach contents $\cdot$ Diet $\cdot$ Resource use

\section{INTRODUCTION}

Invasive species play a major role in humaninduced environmental changes across the planet (Mack et al. 2000). In marine ecosystems, established invasive species pose a major threat to biological diversity (Vitousek et al. 1997) by impacting community structure and function and modifying ecosystem processes, which together may have long-lasting ecological and economic consequences throughout invaded regions (Molnar et al. 2008).

Lionfishes (Pterois volitans and P. miles), native to the Indian and Pacific Oceans, are recognized as the first invasive teleost to expand its range successfully into the Atlantic Ocean (Whitfield et al. 2002). Following their first documented sighting off Dania

\footnotetext{
*Corresponding author: coreyeddy1@gmail.com
}

Point, Florida in 1985, lionfish dispersed rapidly northward along the US coast, through the Caribbean Sea, and into the Gulf of Mexico (Schofield 2010). Ferreira et al. (2015) reported the first capture of an invasive lionfish in Brazil in 2014, an event predicted by their thermal tolerance, generalist feeding behavior, habitat versatility, ability to exploit multiple habitats, and broad latitudinal range in the Indo-Pacific (Morris \& Whitfield 2009, Luiz et al. 2013). Today, lionfish are one of the most common, and oftentimes most abundant mesopredators in parts of their invaded range (Whitfield et al. 2007).

The progression of the lionfish invasion in Bermuda appears to be unique compared to other locations in the northwestern Atlantic. The first lionfish recorded in Bermuda was collected in 2000 from a

(c) C.E., J.P., S.S., G.G.-G., D.B., and the USA Government 2016. Open Access under Creative Commons by Attribution Licence. Use, distribution and reproduction are unrestricted. Authors and original publication must be credited.

Publisher: Inter-Research · www.int-res.com 
tide pool in Devonshire Bay. In the years following, additional lionfish were captured or sighted in low numbers around Bermuda, with only 12 reported between 2001 and 2003, and 18 reported during the summer of 2004 (S. Manuel, Bermuda's Department of Environment and Natural Resources, unpubl. data). Although Bermuda was the first country outside of the USA to report lionfish in the Atlantic, the lionfish population there appears to have grown more slowly than in other regions (C. Eddy unpubl. data). In the Bahamas, for example, lionfish densities reached approximately 400 fish ha ${ }^{-1}$ only 4 yr after their initial invasion began (Green \& Côté 2009), and the mean lionfish density on natural reefs in the northern Gulf of Mexico reached 490 fish ha ${ }^{-1}$ within $3 \mathrm{yr}$ of the first lionfish sighting (Dahl \& Patterson 2014, Frazer et al. 2012). This difference could be attributed to the effects of strong seasonal temperature changes in Bermuda on reproduction (i.e. a relatively shorter spawning season due to cold winter sea temperatures; Morris 2009, Smith et al. 2013), limited recruitment from other regions due to Bermuda's isolation (Schultz \& Cowen 1994), and ocean currents that may advect locally spawned eggs and larvae away from the island (R. Johnson, Bermuda Institute of Ocean Sciences, pers. comm.). Nonetheless, anecdotal evidence and catch data (e.g. public culling efforts and lobster fishery bycatch; Bermuda's Department of Environment and Natural Resources unpubl. data) from Bermuda suggests that the lionfish population continues to expand, and there is concern that Bermuda may still be in the early stages of invasive species population growth, characterized by a period of slow growth followed by a period of exponential growth (Sakai et al. 2001).

As a generalist and opportunistic invasive predator with highly effective and unique hunting strategies (i.e. ambush predation, cooperative hunting, palpation, herding, and directed water-jets) (Kendall 1990, Morris \& Akins 2009, Albins \& Lyons 2012), lionfish consume large quantities and a broad diversity of juvenile and small-bodied adult reef fish as well as small invertebrates (Morris \& Akins 2009). Prey naïveté in the invaded range, along with the lionfishes' resemblance to more benign organisms, also appears to contribute to their hunting success (Cure et al. 2012). Considering these feeding characteristics, their rapid growth rate (Edwards et al. 2014), small size-at-maturity (Morris 2009, Gardner et al. 2015), high fecundity and high spawning frequency (Morris 2009, Gardner et al. 2015), and apparent lack of natural predators (Albins \& Hixon 2013), there is great concern that if their populations are not prop- erly managed, invasive lionfish could cause significant ecological disruption in Bermuda, through predation and resource competition. Elsewhere, Albins \& Hixon (2008) showed that lionfish can reduce the recruitment of reef fish by nearly $80 \%$ in as little as 5 wk. Further, Lesser \& Slattery (2011) suggest that lionfish are the cause of widespread declines of herbivorous reef fish and a subsequent phase shift that occurred at mesophotic depths around the Bahamas from a healthy, robust coral ecosystem to an algaedominated community. Additionally, across study sites in the Bahamas, Green et al. (2012) showed that the biomass of lionfish prey species declined by $65 \%$ between 2008 and 2010, while lionfish biomass simultaneously increased from 23 to $\sim 40 \%$ of total predator biomass. Thus, invasive lionfish can potentially negatively impact ecologically and economically important species, with consequent effects cascading through invaded ecosystems.

To explore the impact lionfish may have upon the coral reef communities of Bermuda, there is a need to understand their location-specific diet. Moreover, to better understand the impacts of these invasive species throughout the invaded range, there is a need to compare their feeding habits in different regions to highlight diet variation and to examine how environmental factors (e.g. depth and water temperature) may alter prey availability, with a subsequent effect on diet. As Bermuda's coral reefs are considered to be some of the healthiest in the Atlantic Ocean (Jackson et al. 2014), this is also an opportunity to study the potential impact of lionfish in an ecosystem with limited impacts from development, pollution, and overfishing. In this study, we describe the diet of Bermuda's invasive lionfish population to provide a more detailed understanding of their potential impact upon the coral reef ecosystem.

\section{MATERIALS AND METHODS}

\section{Collections}

Lionfish were collected from multiple locations around the Bermuda platform $\left(32^{\circ} 21^{\prime} \mathrm{N}, 64^{\circ} 48^{\prime} \mathrm{W}\right)$ between January 2013 and February 2016 (Fig. 1). Specimens $(n=1508)$ were collected by commercial fishermen (lobster traps, $\mathrm{n}=75$ ), recreational fishermen (hook and line, $\mathrm{n}=2$ ), permitted lionfish cullers (pole-spear, $\mathrm{n}=1045$ ), researchers (pole-spear, $\mathrm{n}=$ 141), and fisheries management staff (experimental lionfish traps, $\mathrm{n}=148$; an additional 97 lionfish were delivered to researchers without a label to indicate a 


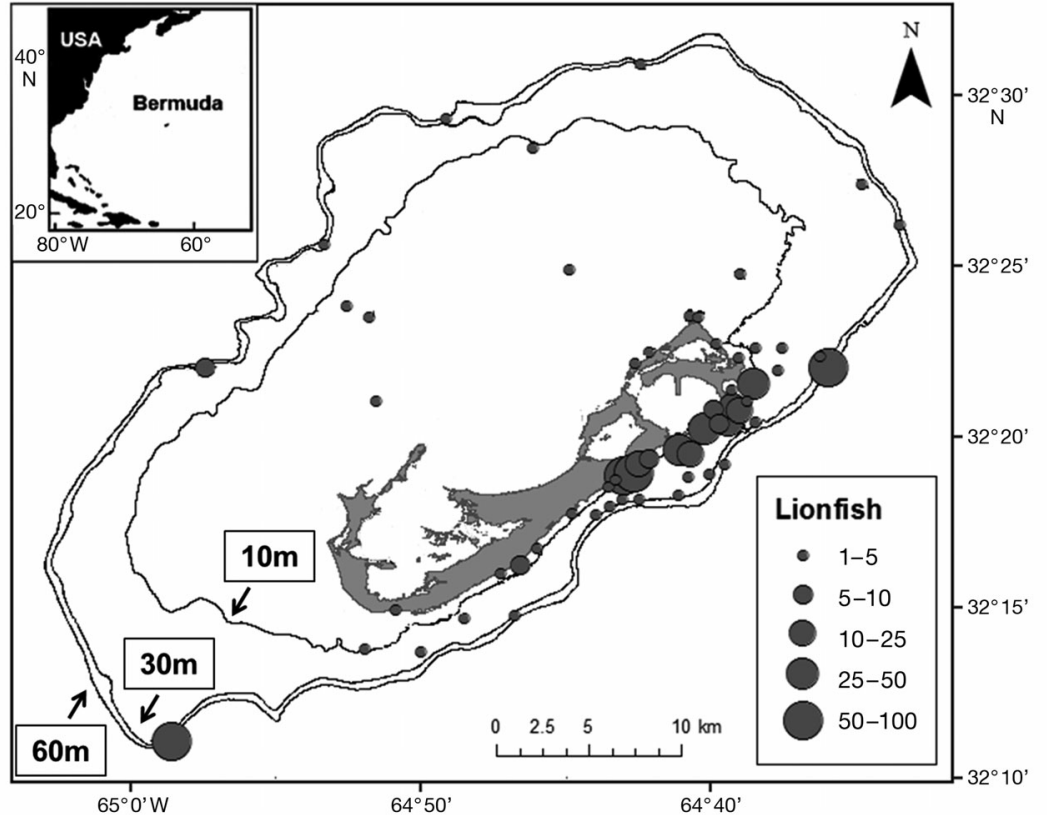

Fig. 1. Locations and relative number of lionfish (Pterois volitans and P. miles) captured between 2013 and 2015 for this study

method). Specimens were collected across a wide range of depths $(0-10 \mathrm{~m}: \mathrm{n}=499 ; 10-20 \mathrm{~m}: \mathrm{n}=148$; 20-30 m: $\mathrm{n}=109 ; 30-40 \mathrm{~m}: \mathrm{n}=19 ; 40-50 \mathrm{~m}: \mathrm{n}=22$; 50-60 m: $\mathrm{n}=415 ;>60 \mathrm{~m}: \mathrm{n}=10$ ). The remaining individuals $(\mathrm{n}=199)$ were provided to the research team with no indication of the depth of capture. Overall, 229 lionfish were captured in 2013, 396 in 2014, 493 in 2015, and 159 in 2016. The remaining individuals ( $n=134$ ) were provided to the research team with no indication of the date of capture. Of those for which we have such data, 325 were captured in winter (January to March), 161 in spring (April to June), 677 in summer (July to September), and 114 in fall (October to December). Prior to dissection, total length (TL), standard length (SL), and body mass were recorded. Lionfish were placed on ice and dissected the same day as they were captured. If dissections had to be delayed, lionfish were frozen at $-20^{\circ} \mathrm{C}$ for the interim.

\section{Stomach contents analyses}

Stomach contents were identified to the lowest taxonomic level possible, counted, and sorted into groups according to their identification. Well-digested prey items that could not be identified to species or family were labeled as 'unidentified' crustacean, crab, lobster, shrimp, or teleost. Occasionally, even this was not possible and items were labeled 'unidentified digested material'. In addition, each prey item was cate- gorized by family and trophic guild (limited to 8 broad categories: cleaner, detritivore, herbivore, invertivore, omnivore, piscivore, planktivore, and zooplanktivore). For each stomach, the mass of each prey group was recorded and volume was measured by water displacement in a graduated cylinder. Neither prey mass nor volume were adjusted for partial digestion, thus these measurements are potentially underestimated. The contribution of each prey taxon to the overall diet of lionfish was quantified using 3 traditional metrics of prey quantity: percent frequency of occurrence $(\% F)$, percent composition by mass $(\% M)$, and percent composition by number $(\% N)$ (Hyslop 1980). For these metrics, omnivorous prey items that belong in multiple trophic guilds were classified as belonging to the guild that best describes the majority of its diet as reported in the literature. To examine the importance of each prey taxon (by species, family, and trophic guild), 3 indices of importance were calculated: the index of relative importance (IRI) (Pinkas et al. 1971), the index of preponderance (IOP) (Natrajan \& Jhingran 1962), and the percent index of relative importance (\%IRI) (Cortés 1997):

$$
\begin{gathered}
\mathrm{IRI}_{a}=F_{a} \times\left(N_{a}+M_{a}\right) \\
\mathrm{IOP}_{a}=\frac{\left(F_{a} \times M_{a}\right)}{\sum_{a=1}^{n}\left(F_{a}+M_{a}\right)} \\
\% \mathrm{IRI}_{a}=100 \times \mathrm{IRI}_{a} \div \sum_{a=1}^{n} \mathrm{IRI}_{a}
\end{gathered}
$$

where $n$ is the number of prey types, $F_{a}$ is the frequency of occurrence of species $a_{1} M_{a}$ is the percent composition by mass of species $a_{1}$ and $N_{a}$ is the percent composition by number of species $a$.

\section{Cumulative prey curve}

A cumulative prey curve was used to assess whether our sample size was sufficient to accurately describe lionfish diet. Identified prey items were grouped by family, and the cumulative number of novel prey items was analyzed using 1000 randomizations of the data (Bizzarro et al. 2007). The mean number of novel prey items for each consecutive stomach $( \pm 95 \%$ confidence interval $)$ 
was calculated, and these values were plotted to create the cumulative prey curve. Sample size sufficiency was assessed using the linear regression method of Bethea et al. (2011), where the slope from a linear regression of the last 4 stomach samples (i.e. an approximation of the rate at which novel prey items are encountered) is compared to a slope of $5 \%$ using a Student's $t$-test of equality of 2 population regression coefficients (Zar 1999). A sufficient sample size is indicated when the slope of the cumulative prey curve's endpoints is not significantly greater than a line with $5 \%$ slope $(p$-value $>\alpha)$. For all statistical tests, we used a significance value of $\alpha=0.05$.

\section{Environmental factors}

Canonical correspondence analysis (CCA) was used to investigate how diet was influenced by environmental variables (e.g. lionfish size, depth, season, and year of capture). The analysis was performed using the ' $\mathrm{cca}$ ' script, available in the software package 'vegan' in R. We used 999 Monte Carlo permutations to evaluate the statistical significance of these explanatory variables in the ordination (ter Braak 1986). CCA examines the multivariate relationship between explanatory variables (e.g. environmental factors) and the weighted average of response variables (i.e. prey items) using a redundancy analysis, based on proportions and sample size (ter Braak 1986, 1987). Environmental variables that do not contribute significantly to diet variation are removed and the remaining relationships are expressed in reduced canonical space (ter Braak 1986, Jongman et al. 1987). CCA can be used to show how the change in environmental variables may affect the distribution of species at specific sites. When used with diet data, CCA explains how the distribution of prey items (i.e. 'species') collected in lionfish stomachs (i.e. 'sites') changes along gradients of these environmental factors, and can highlight the extent to which each drives diet variance. Using prey-environment bi-plots, CCA results are best understood by highlighting the amount of variance explained by each canonical axis and examining the correlation between the canonical axes and the explanatory variables (ter Braak 1986, 1987). The response variables were the prey items' contribution to lionfish diet by mass (i.e. $\% M$ ), and the data were $\ln (x+1)$-transformed to account for positive skewness.

\section{RESULTS}

Collected lionfish $(\mathrm{n}=1508)$ ranged in size from 124 to $467 \mathrm{~mm}$ TL (mean \pm SE: $335 \pm 2 \mathrm{~mm}$ ). A total of 2703 prey items were removed from 1352 stomachs and assigned to the lowest possible taxonomic level. The mass of prey by taxon was measured for 818 stomachs. Overall prey items were removed from a broad size-range of lionfish $(100-150 \mathrm{~mm}: \mathrm{n}=9 ; 150$ $200 \mathrm{~mm}: \mathrm{n}=23 ; 200-250 \mathrm{~mm}: \mathrm{n}=66 ; 250-300 \mathrm{~mm}$ : $\mathrm{n}=240 ; 300-350 \mathrm{~mm}: \mathrm{n}=396 ; 350-400 \mathrm{~mm}: \mathrm{n}=403$; $400-450 \mathrm{~mm}: \mathrm{n}=207 ;>450 \mathrm{~mm}: \mathrm{n}=8$ ). The number of prey items per stomach ranged from 0 to 27 (mean \pm SE: $2.0 \pm 0.08$ ). A total of 15 stomachs were everted, while 141 stomachs had been removed prior to donation to the research team. In total, $36.0 \%$ of stomachs ( $\mathrm{n}=487$ ) were empty. Overall, $28.1 \%$ of stomachs from lionfish captured in shallow water $(<30 \mathrm{~m})$ were empty, as were $61.6 \%$ of stomachs from lionfish captured in deep water $(>30 \mathrm{~m})$.

The cumulative prey curve suggests that a sufficient number of stomachs was analyzed to provide an accurate description of lionfish diet (slope $<5 \%$; $\mathrm{p}=$ 0.04) (Fig. 2). There were 91 novel prey groups identified and, on average, 3 novel items were collected from the final 79 stomachs. Although rare prey items found in only a single stomach seem to prevent the cumulative prey curve from reaching a true asymptote, as originally described by Bizzarro et al. (2007), we examined more stomachs than similar studies (e.g. Morris \& Akins 2009) and found a broadly similar prey base, so we feel confident that our results accurately describe the diet of lionfish in Bermuda.

\section{Prey composition}

In total, 22 families of teleosts, 14 families of crustaceans, and 3 families of mollusks contributed to the diet of lionfish (Table 1). Teleosts accounted for $55.5 \%$ of the lionfish diet by number $(\% N), 73.4 \%$ by mass $(\% M)$, and occurred in $51.9 \%$ of stomachs $(\% F)$. Other prey items included crustaceans $(43.0 \% N$, $23.4 \% M, 33.8 \% F)$, mollusks $(0.2 \% N, 1.1 \% M, 0.4 \% F)$, and unidentified prey items $(3.6 \% N, 2.1 \% M, 6.9 \% F)$.

Teleost prey included 44 identifiable species (Table 1). The most speciose families were Labridae (6 spp.), Monocanthidae (5 spp.), Serranidae (4 spp.), Apogonidae (4 spp.), and Pomacentridae (4 spp.). The 5 most common teleost prey families accounted for $20 \%$ of the overall diet by number and $66 \%$ of all identifiable teleosts items (Labridae: $7.0 \% N_{i}$ Blennidae: $3.7 \% N_{i}$ Holocentridae: $3.6 \% N_{i}$ Scaridae: $2.8 \% N_{i}$ 


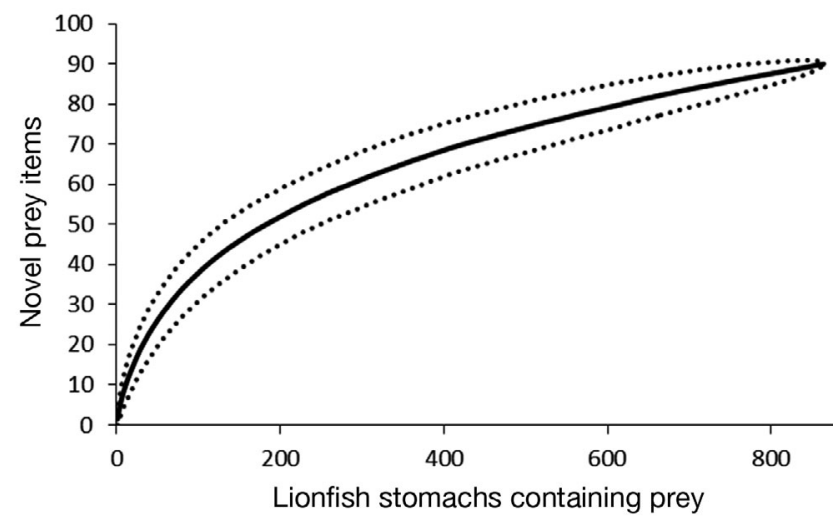

Fig. 2. Mean cumulative number of prey taxa per lionfish (Pterois volitans and $P$. miles) stomach sample $\pm 95 \%$ confidence interval (dotted lines)

and Serranidae: $2.5 \% N$ ). By mass, the 5 most common prey families accounted for $34 \%$ of the overall diet and $71 \%$ of all identifiable teleosts (Labridae: $10.7 \% M$; Holocentridae: $8.6 \% M$; Serranidae: $6.1 \% M$; Blennidae: $4.8 \% M$; and Haemulidae: $4.1 \% M$ ). The 5 families that occurred most frequently in lionfish stomachs included Labridae $(6.6 \% F)$, Blennidae $(3.2 \% F)$, Holocentridae $(3.1 \% F)$, Serranidae $(2.3 \% F)$, and Gobiidae $(2.1 \% F)$.

Crustacean prey included 17 species (Table 1). The most speciose families were Calappidae (3 spp.) and Grapsidae (3 spp.). The 5 most common crustacean prey families accounted for $28 \%$ of the overall diet by number and $97 \%$ of all identifiable crustaceans (Rhynchocinetidae: $13.7 \% N_{\text {; }}$ Munididae: $6.2 \% N_{i} \mathrm{Ca}-$ lappidae: $4.0 \% N_{i}$ Portunidae: $2.6 \% N_{i}$ and Grapsidae: $1.4 \% N$ ). By mass, the 5 most common prey families accounted for $20 \%$ of the overall diet and $96 \%$ of all identifiable crustaceans (Rhynchocinetidae: 14.0\% $M$; Grapsidae: 2.6\% $M$; Calappidae: $1.4 \% M$; Mithracidae: $1.0 \% M$; and Munididae: $0.6 \% M$ ). The 5 families that occurred most frequently included Rhynchocinetidae $(11.1 \% F)$, Munididae $(3.3 \% F)$, Portunidae $(1.8 \% F)$, Calappidae $(1.4 \% F)$, and Grapsidae $(1.3 \% F)$.

The red night shrimp Cinetorhynchus rigens, also known as the mechanical shrimp, was the most common prey item across all metrics $(13.7 \% N, 14.0 \% M$, and $11.1 \% F$ ) (Table 2). Depending upon the metric in use, the second most common species was either squat lobster Munida simplex $(6.2 \% N)$ or bluehead wrasse Thalassoma bifasciatum ( $7.6 \% M$ and $3.4 \% F)$. The third most common species also differed by diet metric and was either the bluehead wrasse $(3.5 \% N)$, reef squirrelfish Sargocentron coruscum $(4.7 \% M)$ or squat lobster $(3.3 \% F)$.

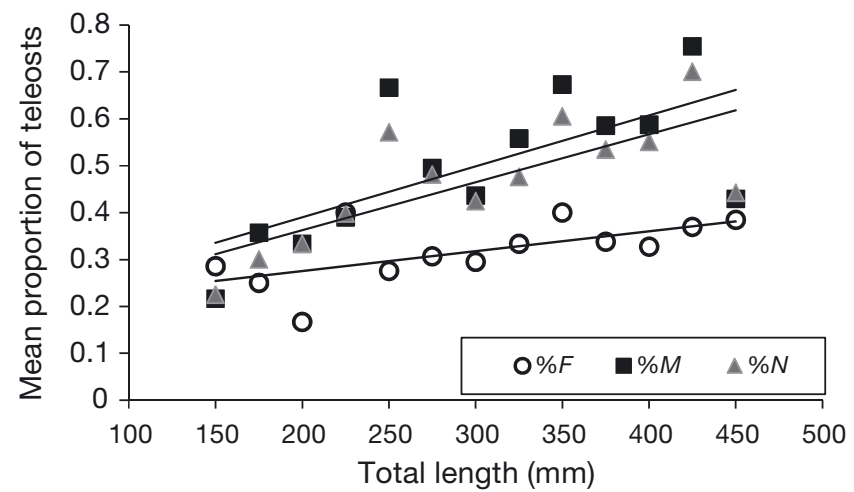

Fig. 3. Mean proportion of teleosts (MPT) in the diet of Bermuda lionfish (Pterois volitans and P. miles), separated into $25 \mathrm{~mm}$ size classes (total length, TL), as described by the 3 common diet metrics - percent composition by number $(\% N)$, percent frequency of occurrence $(\% F)$, and percent composition by mass $(\% M)$. Lines indicate a significant increase in the MPT with lionfish TL: $\mathrm{MPT}_{\% F}=0.0004(\mathrm{TL})+0.1912 ; \mathrm{MPT}_{\% M}=$ $0.0011(\mathrm{TL})+0.1728 ; \mathrm{MPT}_{\% N}=0.001(\mathrm{TL})+0.1584$

The contribution of teleosts within the lionfish diet increased significantly with lionfish size across all 3 diet metrics $\left(\% N\right.$ : $\mathrm{R}^{2}=0.5714, F_{1,13}=13.96, \mathrm{p}<0.05$; $\% M: \mathrm{R}^{2}=0.4615, F_{1,13}=9.65, \mathrm{p}<0.05 ; \% F: \mathrm{R}^{2}=$ 0.3883, $F_{1,13}=6.40, \mathrm{p}<0.05$ ) (Fig. 3).

\section{Indices of importance}

In both \%IRI and IOP, the top-ranked prey items, both by species and family, were relatively consistent (Table 3). Two species (red night shrimp and bluehead wrasse) and their corresponding 2 families (Rhynchocinetidae and Labridae) were ranked highest, suggesting they make a substantial and important contribution to the diet of lionfish in Bermuda. Nine species and 8 families were consistently ranked within the top 10 prey items (Table 3 ). There was little variation in the relative importance of prey items characterized by trophic position, with invertivores, detrivores, and herbivores consistently ranked highest (Table 3).

\section{Environmental factors}

Eigenvalues for the 4 multivariate axes were 0.49 (CCA1), 0.31 (CCA2), 0.23 (CCA3), and 0.14 (CCA4). Depth, season, and lionfish size significantly influence the diet of lionfish in Bermuda and generally correspond to CCA1 ( $p=0.001), \operatorname{CCA} 2$ ( $p=0.001)$, and CCA3 $(p=0.006)$, respectively. CCA1 and CCA2 (Fig. 4a) accounted for 42.1 and $26.6 \%$ of the explain- 
Table 1. Contribution of lionfish (Pterois volitans and P. miles) prey by taxa, showing percent composition by number (\% $N$ ), percent composition by mass $(\% M)$, and percent frequency of occurrence $(\% F)$. C: cleaner; D: detritivore; H: herbivore; I: invertivore; O: omnivore; P: piscivore; Pl: planktivore; Z: zooplanktivore. Trophic guild information collected from Bohlke \& Chaplin (1993), Cartes (1993), Cervigón (1993), Chande \& Mgaya (2005), Corredor (1978), Frick et al. (2004), Hughes \& Elner (1989), Iversen et al. (1986), Lavalli et al. (2007), McEachran (2009), Puccio et al. (2006), Randall (1967), Robertson (1981), Romero et al. (2004), Ryan (1956), Samson et al. (2007), Sterrer (1986, 1992), Whiteman et al. (2007), and Zhang et al. (1998)

\begin{tabular}{|c|c|c|c|c|c|}
\hline Taxon & $\begin{array}{l}\text { Frequency } \\
\text { (stomachs) }\end{array}$ & $\begin{array}{c}\% N \\
(\mathrm{n}=1352)\end{array}$ & $\begin{array}{c}\% M \\
(\mathrm{n}=818)\end{array}$ & $\begin{array}{c}\% F \\
(\mathrm{n}=1352)\end{array}$ & $\begin{array}{l}\text { Trophic } \\
\text { guild }\end{array}$ \\
\hline $\begin{array}{l}\text { Unidentified } \\
\text { Mollusca }\end{array}$ & 96 & 3.6 & 2.1 & 6.9 & - \\
\hline \multicolumn{6}{|l|}{$\begin{array}{l}\text { Mollusca } \\
\text { Loliginidae }\end{array}$} \\
\hline Sepioteuthis sepioidea & 2 & 0.1 & 0.9 & 0.1 & $\mathrm{P}$ \\
\hline \multicolumn{6}{|l|}{ Octopodidae } \\
\hline Octopus spp. & 2 & 0.1 & 0.2 & 0.1 & I \\
\hline \multicolumn{6}{|l|}{ Marginellidae } \\
\hline Volvarina albolineata $^{\mathrm{b}}$ & 1 & $<0.1$ & $<0.1$ & 0.1 & $\mathrm{Pl}, \mathrm{Z}$ \\
\hline TOTAL & 5 & 0.2 & 1.1 & 0.4 & \\
\hline \multicolumn{6}{|l|}{ Crustacea } \\
\hline Unidentified crustacean & 5 & 0.2 & $<0.1$ & 0.4 & - \\
\hline Unidentified shrimp & 155 & 12.6 & 2.8 & 11.1 & - \\
\hline Unidentified crab & 28 & 1.7 & 0.2 & 2.0 & - \\
\hline Unidentified lobster & 1 & $<0.1$ & $<0.1$ & 0.1 & - \\
\hline \multicolumn{6}{|l|}{ Rhynchocinetidae } \\
\hline Cinetorhynchus rigens & 155 & 13.7 & 14.0 & 11.1 & $\mathrm{D}$ \\
\hline \multicolumn{6}{|l|}{ Munididae } \\
\hline Munida simplex & 46 & 6.2 & 0.6 & 3.3 & $\mathrm{D}, \mathrm{H}, \mathrm{I}$ \\
\hline \multicolumn{6}{|l|}{ Portunidae } \\
\hline Portunus anceps & 26 & 2.6 & 0.2 & 1.9 & $\mathrm{H}, \mathrm{I}$ \\
\hline \multicolumn{6}{|l|}{ Grapsidae } \\
\hline Percnon gibbesi & 15 & 1.3 & 2.6 & 1.1 & $\mathrm{H}$ \\
\hline Plagusia depressa & 1 & $<0.1$ & $<0.1$ & 0.1 & $\mathrm{H}$ \\
\hline Planes minutus & 2 & 0.1 & $<0.1$ & 0.1 & $\mathrm{O}$ \\
\hline Calappidae $^{\mathrm{a}}$ & 7 & 3.0 & 0.1 & $<0.1$ & $\mathrm{I}$ \\
\hline Cryptosoma bairdii & 16 & 0.9 & 1.2 & 1.1 & I \\
\hline Calappa gallus & 2 & 0.1 & 0.1 & 0.1 & I \\
\hline Calappa ocellata & 1 & $<0.1$ & $<0.1$ & 0.1 & I \\
\hline \multicolumn{6}{|l|}{ Stenopodidae } \\
\hline Stenopus hispidus & 5 & 0.2 & 0.2 & 0.4 & $\mathrm{C}$ \\
\hline \multicolumn{6}{|l|}{ Hippolytidae } \\
\hline Lysmata grabhami & 3 & 0.1 & $<0.1$ & 0.2 & $\mathrm{C}$ \\
\hline \multicolumn{6}{|l|}{ Lysiosquillidae } \\
\hline Lysiosquilla scabricauda & 2 & 0.1 & 0.2 & 0.1 & I, P \\
\hline \multicolumn{6}{|l|}{ Gonodactylidae } \\
\hline Neogonodactylus oerstedii & 1 & $<0.1$ & 0.1 & 0.1 & I \\
\hline \multicolumn{6}{|l|}{ Palaemonidae } \\
\hline Brachycarpus biunguiculatus & 1 & $<0.1$ & 0.1 & 0.1 & $\mathrm{C}$ \\
\hline Palaemon northropi & 1 & 0.1 & $<0.1$ & 0.1 & $\mathrm{D}$ \\
\hline \multicolumn{6}{|l|}{ Mithracidae } \\
\hline Mithraculus forceps & 3 & 0.1 & 0.9 & 0.2 & $\mathrm{D}$ \\
\hline Penaeidae & 1 & 0.1 & $<0.1$ & 0.1 & I \\
\hline \multicolumn{6}{|l|}{ Scyllaridae } \\
\hline Scyllarides nodifer & 1 & $<0.1$ & 0.1 & 0.1 & I \\
\hline Xanthidae & 1 & $<0.1$ & $<0.1$ & 0.1 & I \\
\hline TOTAL & 479 & 43.0 & 23.4 & 33.8 & \\
\hline \multicolumn{6}{|l|}{ Teleosts } \\
\hline Unidentified teleost & 326 & 25.6 & 25.3 & 23.4 & - \\
\hline Labridae $^{\mathrm{a}}$ & 9 & 0.6 & 0.2 & 0.6 & - \\
\hline Thalassoma bifasciatum & 48 & 3.5 & 7.6 & 3.4 & $\mathrm{C}, \mathrm{Z}$ \\
\hline Halichoeres garnoti & 15 & 1.4 & 0.7 & 1.1 & I, P \\
\hline Halichoeres bivittatus & 15 & 1.3 & 2.2 & 1.1 & I, P \\
\hline Xyrichtys martinicensis & 3 & 0.1 & $<0.1$ & 0.2 & I \\
\hline Halichoeres maculipinna & 1 & $<0.1$ & $<0.1$ & 0.1 & I, P \\
\hline Clepticus parrae & 1 & $<0.1$ & $<0.1$ & 0.1 & $\mathrm{Pl}, \mathrm{Z}$ \\
\hline \multicolumn{6}{|l|}{ Serranidae } \\
\hline Paranthias furcifer & 24 & 2.1 & 4.5 & 1.7 & $\mathrm{Z}$ \\
\hline Liopropoma spp. & 3 & 0.1 & 0.4 & 0.2 & I, P \\
\hline
\end{tabular}


Table 1 (continued)

\begin{tabular}{|c|c|c|c|c|c|}
\hline Taxon & $\begin{array}{l}\text { Frequency } \\
\text { (stomachs) }\end{array}$ & $\begin{array}{c}\% N \\
(\mathrm{n}=1352)\end{array}$ & $\begin{array}{c}\% M \\
(\mathrm{n}=818)\end{array}$ & $\begin{array}{c}\% F \\
(\mathrm{n}=1352)\end{array}$ & $\begin{array}{l}\text { Trophic } \\
\text { guild }\end{array}$ \\
\hline Hypoplectrus puella & 4 & 0.2 & 1.0 & 0.3 & I, P \\
\hline Liopropoma rubre & 1 & $<0.1$ & 0.3 & 0.1 & $\mathrm{I}, \mathrm{P}$ \\
\hline Holocentridae $^{\mathrm{a}}$ & 26 & 1.9 & 3.2 & 1.9 & \\
\hline Sargocentron coruscum & 13 & 1.5 & 4.7 & 0.9 & I \\
\hline Holocentrus adscensionis & 1 & $<0.1$ & 0.4 & 0.1 & $\mathrm{I}$ \\
\hline Sargocentron vexillarium & 3 & 0.1 & 0.3 & 0.2 & $\mathrm{I}, \mathrm{P}$ \\
\hline Bothidae & 16 & 1.3 & 1.4 & 1.1 & I, P \\
\hline Gobiidae $^{a}$ & 3 & 0.2 & 0.1 & 0.2 & \\
\hline Coryphopterus glaucofraen & um 18 & 1.4 & 1.1 & 1.3 & $\mathrm{D}, \mathrm{H}, \mathrm{I}$ \\
\hline Coryphopterus personatus & 4 & 0.2 & 0.1 & 0.3 & $\mathrm{Pl}$ \\
\hline Gnatholepis thompsoni & 4 & 0.3 & 0.3 & 0.3 & $\mathrm{D}, \mathrm{H}, \mathrm{I}$ \\
\hline Scaridae $^{\mathrm{a}}$ & 12 & 1.7 & 0.6 & 0.9 & \\
\hline Sparisoma aurofrenatum & 11 & 1.0 & 0.2 & 0.8 & $\mathrm{H}$ \\
\hline Scarus taeniopterus & 4 & 0.1 & 0.4 & 0.3 & $\mathrm{H}$ \\
\hline Blennidae $^{\mathrm{a}}$ & 13 & 1.4 & 1.2 & 0.9 & \\
\hline Parablennius marmoreus & 20 & 1.2 & 2.2 & 1.4 & $\mathrm{D}, \mathrm{H}, \mathrm{I}, \mathrm{Pl}$ \\
\hline Entomacrodus nigricans & 12 & 1.2 & 1.4 & 0.9 & $\mathrm{D}, \mathrm{H}, \mathrm{Pl}$ \\
\hline Haemulidae $^{\mathrm{a}}$ & 10 & 1.0 & 0.8 & 0.7 & \\
\hline Haemulon aurolineatum & 5 & 0.6 & 0.2 & 0.4 & I \\
\hline Haemulon flavolineatum & 8 & 0.7 & 3.0 & 0.6 & I \\
\hline Apogonidae $^{a}$ & 11 & 0.5 & 0.9 & 0.8 & \\
\hline Apogon pseudomaculatus & 1 & $<0.1$ & 0.2 & 0.1 & $\mathrm{I}, \mathrm{Z}$ \\
\hline Apogon townsendi & 1 & $<0.1$ & 0.1 & 0.1 & $\mathrm{I}, \overline{\mathrm{Z}}$ \\
\hline Apogon binotatus & 1 & $<0.1$ & 0.1 & 0.1 & I, Z \\
\hline Apogon maculatus & 3 & 0.1 & 0.3 & 0.2 & I, Z \\
\hline Chaetodontidae $^{\mathrm{a}}$ & 7 & 0.4 & 0.1 & 0.5 & \\
\hline Chaetodon ocellatus & 6 & 0.3 & 0.4 & 0.4 & I \\
\hline Chaetodon capistratus & 2 & 0.2 & $<0.1$ & 0.1 & I \\
\hline Acanthuridae & 7 & 0.3 & 0.1 & 0.5 & \\
\hline Acanthurus chirurgus & 1 & $<0.1$ & 0.1 & 0.1 & $\mathrm{H}$ \\
\hline Acanthurus bahianus & 4 & 0.2 & 0.5 & 0.3 & $\mathrm{H}$ \\
\hline Synodontidae $^{\mathrm{a}}$ & 4 & 0.2 & 0.1 & 0.3 & \\
\hline Synondus synodus & 2 & 0.1 & 0.2 & 0.1 & $\mathrm{P}, \mathrm{Z}$ \\
\hline \multicolumn{6}{|l|}{ Aulostomidae } \\
\hline Aulostomus maculatus & 4 & 0.1 & $<0.1$ & 0.3 & I, P \\
\hline Mullidae $^{\mathrm{a}}$ & 1 & $<0.1$ & 0.5 & 0.1 & \\
\hline Pseudupeneus maculatus & 14 & 0.7 & 3.3 & 1.0 & I \\
\hline Mulloidichthys martinicus & 1 & $<0.1$ & 0.2 & 0.1 & I \\
\hline \multicolumn{6}{|l|}{ Pempheridae } \\
\hline Pempheris schomburgkii & 2 & 0.3 & 0.4 & 0.1 & I, Z \\
\hline \multicolumn{6}{|l|}{ Pomacentridae } \\
\hline Stegastes variabilis & 1 & $<0.1$ & 0.2 & 0.1 & $\mathrm{D}, \mathrm{H}, \mathrm{I}$ \\
\hline Stegastes spp. & 3 & 0.1 & 0.2 & 0.2 & $\mathrm{D}, \mathrm{H}, \mathrm{I}$ \\
\hline Chromis insolata & 1 & $<0.1$ & 0.1 & 0.1 & $\mathrm{Z}$ \\
\hline Chromis flavicauda & 1 & 0.1 & $<0.1$ & 0.1 & $\mathrm{Z}$ \\
\hline \multicolumn{6}{|l|}{ Monocanthidae } \\
\hline Cantherhines spp. & 1 & $<0.1$ & $<0.1$ & 0.1 & $\mathrm{H}, \mathrm{I}$ \\
\hline Aluterus schoepfii & 1 & $<0.1$ & $<0.1$ & 0.1 & $\mathrm{H}$ \\
\hline Monacanthus tuckeri & 4 & 0.2 & 1.0 & 0.3 & $\mathrm{I}, \mathrm{Pl}$ \\
\hline Monacanthus ciliatus & 1 & $<0.1$ & $<0.1$ & 0.1 & $\mathrm{H}, \mathrm{I}, \mathrm{Pl}$ \\
\hline Aluterus scriptus & 1 & $<0.1$ & 0.1 & 0.1 & $\mathrm{H}, \mathrm{I}$ \\
\hline \multicolumn{6}{|l|}{ Carangidae } \\
\hline Decapterus spp. & 1 & $<0.1$ & 0.2 & 0.1 & $\mathrm{Z}$ \\
\hline \multicolumn{6}{|l|}{ Sparidae } \\
\hline Diplodus bermudensis & 1 & $<0.1$ & $<0.1$ & 0.1 & $\mathrm{H}$ \\
\hline \multicolumn{6}{|l|}{ Clupeidae } \\
\hline Jenkinsia lamprotaenia & 1 & $<0.1$ & $<0.1$ & 0.1 & $\mathrm{Z}$ \\
\hline Antennariidae & 1 & $<0.1$ & $<0.1$ & 0.1 & $\mathrm{I}, \mathrm{P}$ \\
\hline \multicolumn{6}{|l|}{ Tetraodontidae } \\
\hline Sphoeroides spengleri & 1 & 0.1 & 0.2 & 0.1 & I \\
\hline TOTAL & 724 & 55.5 & 73.4 & 51.9 & \\
\hline
\end{tabular}


Table 2. Top 10 prey species of lionfish (Pterois volitans and P. miles) in Bermuda according to the relative metrics of prey quantity: percent composition by number $(\% N)$, percent composition by mass $(\% M)$, and percent frequency of occurrence $(\% F) .{ }^{\mathrm{A}}=$ crustacean; $^{\mathrm{B}}=$ teleost

\begin{tabular}{|c|c|c|c|c|c|c|}
\hline Rank & Species & $\% N$ & Species & $\% M$ & Species & $\% F$ \\
\hline 1 & Cinetorhynchus rigens ${ }^{\mathrm{A}}$ & 13.7 & Cinetorhynchus rigens ${ }^{\mathrm{A}}$ & 14.0 & Cinetorhynchus rigens ${ }^{\mathrm{A}}$ & 11.1 \\
\hline 2 & Munida simplex ${ }^{\mathrm{A}}$ & 6.2 & Thalassoma bifasciatum ${ }^{\mathrm{B}}$ & 7.6 & Thalassoma bifasciatum ${ }^{\mathrm{B}}$ & 3.4 \\
\hline 3 & Thalassoma bifasciatum $^{\mathrm{B}}$ & 3.5 & Sargocentron coruscum ${ }^{\mathrm{B}}$ & 4.7 & Munida simplex ${ }^{\mathrm{A}}$ & 3.3 \\
\hline 4 & Portunus anceps ${ }^{\mathrm{A}}$ & 2.6 & Paranthias furcifer ${ }^{\mathrm{B}}$ & 4.5 & Portunus anceps ${ }^{\mathrm{A}}$ & 1.9 \\
\hline 5 & Paranthias furcifer ${ }^{\mathrm{B}}$ & 2.1 & Pseudupeneus maculatus ${ }^{\mathrm{B}}$ & 3.3 & Paranthias furcifer ${ }^{\mathrm{B}}$ & 1.7 \\
\hline 6 & Sargocentron coruscum ${ }^{\mathrm{B}}$ & 1.5 & Haemulon flavolineatum ${ }^{\mathrm{B}}$ & 3.0 & Parablennius marmoreus ${ }^{\mathrm{B}}$ & 1.4 \\
\hline 7 & Coryphopterus glaucofraenum ${ }^{\mathrm{B}}$ & 1.4 & Percnon gibbesi ${ }^{\mathrm{A}}$ & 2.6 & Coryphopterus glaucofraenum ${ }^{\mathrm{B}}$ & 1.3 \\
\hline 8 & Halichoeres garnoti $^{\mathrm{B}}$ & 1.4 & Halichoeres bivittatus ${ }^{\mathrm{B}}$ & 2.2 & Cryptosoma bairdii ${ }^{\mathrm{A}}$ & 1.1 \\
\hline 9 & Percnon gibbesi ${ }^{\mathrm{A}}$ & 1.3 & Parablennius marmoreus ${ }^{\mathrm{B}}$ & 2.2 & Percnon gibbesi ${ }^{\mathrm{A}}$ & 1.1 \\
\hline 10 & Halichoeres bivittatus ${ }^{\mathrm{B}}$ & 1.3 & Entomacrodus nigricans ${ }^{\mathrm{B}}$ & 1.4 & Halichoeres garnoti ${ }^{\mathrm{B}}$ & 1.1 \\
\hline
\end{tabular}

able diet variance, respectively, while CCA3 (Fig. 4b) accounted for $19.2 \%$.

\section{DISCUSSION}

In general, the diet of lionfish in Bermuda appears to be broadly similar to that of lionfish found in other locations around the world, consisting of a diverse range of teleost and crustacean prey. However, compared other parts of its invaded range, the contribution of teleosts by number $(\% N)$ to the diet of lionfish in Bermuda appears to be $\sim 15$ to $30 \%$ lower
$(55.5 \% N)$ than other regions $(71.2 \% N$ in the $\mathrm{Ba}-$ hamas, $74.4 \% N$ in the Mexican Caribbean, and $84.1 \% N$ along the southeastern US coast; Morris \& Akins 2009, Muñoz et al. 2011, Valdez-Moreno et al. 2012). In addition, crustaceans appear to play a larger role in the diet of lionfish in Bermuda $(43.0 \% N)$ relative to the Bahamas $(28.5 \% N)$, the Mexican Caribbean $(25.6 \% N)$, and the southeastern US coast $(13.9 \% N)$ (Table 4). The greater contribution of teleost prey items by mass $(73.4 \% M)$ suggests that they make a more important contribution to the diet in terms of energy and nutrients compared to crustaceans $(23.4 \% M)$. This scenario is similar to that

Table 3. Top lionfish (Pterois volitans and P. miles) prey species, families, and trophic guilds in Bermuda according to index of preponderance (IOP) and percent index of relative importance (\%IRI) (values shown in parentheses). ${ }^{\mathrm{A}}=$ crustacean $^{\mathrm{B}}=$ teleost

\begin{tabular}{|c|c|c|c|c|c|c|}
\hline \multirow[t]{2}{*}{ Rank } & \multicolumn{2}{|c|}{ Species } & \multicolumn{2}{|l|}{ - Family } & \multicolumn{2}{|c|}{$\ldots$ Trophic guild } \\
\hline & IOP & $\%$ IRI & IOP & $\%$ IRI & IOP & \%IRI \\
\hline 1 & $\begin{array}{c}\text { Cinetorhynchus rigens }{ }^{\mathrm{A}} \\
(0.81)\end{array}$ & $\begin{array}{c}\text { Cinetorhynchus rigens }{ }^{\mathrm{A}} \\
\text { (69.3) }\end{array}$ & $\begin{array}{c}\text { Rhynchocinetidae } \\
\text { (0.81) }\end{array}$ & $\begin{array}{l}\text { Rhynchocinetidae } \\
\text { (52.0) }\end{array}$ & $\begin{array}{c}\text { Invertivore } \\
\text { (1.99) }\end{array}$ & $\begin{array}{l}\text { Invertivore } \\
\quad(60.4)\end{array}$ \\
\hline 2 & $\begin{array}{c}\text { Thalassoma bifasciatum } \\
\text { (0.14) }\end{array}$ & $\begin{array}{c}\text { Thalassoma bifasciatum } \\
\text { (8.6) }\end{array}$ & $\begin{array}{c}\text { Labridae }^{B} \\
(0.37)\end{array}$ & $\begin{array}{l}\text { Labridae }^{\mathrm{B}} \\
\quad(19.8)\end{array}$ & $\begin{array}{c}\text { Detrivore } \\
(0.89)\end{array}$ & $\begin{array}{c}\text { Detrivore } \\
(27.3)\end{array}$ \\
\hline 3 & $\begin{array}{c}\text { Paranthias furcifer } \\
\text { (0.04) }\end{array}$ & Munida simplex ${ }^{\mathrm{A}}$ & $\begin{array}{c}\text { Holocentridae }^{\mathrm{B}} \\
(0.14)\end{array}$ & $\begin{array}{c}\text { Holocentridae }^{B} \\
(6.3)\end{array}$ & $\begin{array}{l}\text { Herbivore } \\
(0.25)\end{array}$ & $\begin{array}{l}\text { Herbivore } \\
(6.9)\end{array}$ \\
\hline 4 & $\begin{array}{c}\text { Sargocentron coruscum }^{\mathrm{B}} \\
(0.02)\end{array}$ & $\begin{array}{c}\text { Paranthias furcifer } \\
\text { (2.6) }\end{array}$ & $\begin{array}{l}\text { Blennidae } \\
(0.08)\end{array}$ & Blennidae ${ }^{\mathrm{B}}$ & Cleaner & $\begin{array}{c}\text { Cleaner } \\
(4.0)\end{array}$ \\
\hline 5 & Pseudupeneus maculatus $^{\mathrm{B}}$ & $\begin{array}{c}\text { Sargocentron coruscum } \\
\text { B }\end{array}$ & $\begin{array}{c}\text { Serranidae }^{B} \\
(0.07)\end{array}$ & Munididae $^{\mathrm{A}}$ & $\begin{array}{c}\text { Zooplanktivore } \\
(0.06)\end{array}$ & $\begin{array}{c}\text { Zooplanktivore } \\
(1.4)\end{array}$ \\
\hline 6 & $\begin{array}{c}\text { Parablennius marmoreus }^{\mathrm{B}} \\
(0.02)\end{array}$ & $\begin{array}{l}\text { Portunus anceps } \\
\text { (1.2) }\end{array}$ & Haemulidae $^{\mathrm{B}}$ & $\begin{array}{c}\text { Serranidae }^{B} \\
(3.4)\end{array}$ & $\begin{array}{l}\text { Planktivore } \\
(<0.01)\end{array}$ & $\begin{array}{c}\text { Planktivore } \\
(0.1)\end{array}$ \\
\hline 7 & $\begin{array}{c}\text { Percnon gibbesi }{ }^{\mathrm{A}} \\
(0.02)\end{array}$ & $\begin{array}{c}\text { Parablennius marmoreus } \\
\text { (1.1) }\end{array}$ & $\begin{array}{l}\text { Mullidae }^{\mathrm{B}} \\
(0.02)\end{array}$ & Haemulidae $^{\mathrm{B}}$ & $\begin{array}{l}\text { Piscivore } \\
(<0.01)\end{array}$ & $\begin{array}{l}\text { Piscivore } \\
(<0.1)\end{array}$ \\
\hline 8 & $\begin{array}{c}\text { Halichoeres bivittatus }{ }^{\mathrm{B}} \\
(0.01)\end{array}$ & $\begin{array}{c}\text { Percnon gibbesi }{ }^{\mathrm{A}} \\
\text { (1.0) }\end{array}$ & $\begin{array}{c}\text { Grapsidae }^{\mathrm{A}} \\
(0.02)\end{array}$ & $\begin{array}{c}\text { Scaridae }^{B} \\
(1.3)\end{array}$ & & \\
\hline 9 & $\begin{array}{c}\text { Munida simplex } \\
(0.01)\end{array}$ & $\begin{array}{c}\text { Pseudupeneus maculatus }{ }^{\mathrm{B}} \\
(0.9)\end{array}$ & $\begin{array}{c}\text { Gobiidae }^{B} \\
(0.02)\end{array}$ & $\begin{array}{c}\text { Gobiidae }^{B} \\
(1.3)\end{array}$ & & \\
\hline 10 & $\begin{array}{c}\text { Haemulon flavolineatum } \\
\text { (0.01) }\end{array}$ & $\begin{array}{c}\text { Halichoeres bivittatus } \\
(0.9)\end{array}$ & $\begin{array}{l}\text { Scaridae }^{B} \\
(0.01)\end{array}$ & $\begin{array}{c}\text { Calappidae } \\
\text { (1.3) }\end{array}$ & & \\
\hline
\end{tabular}



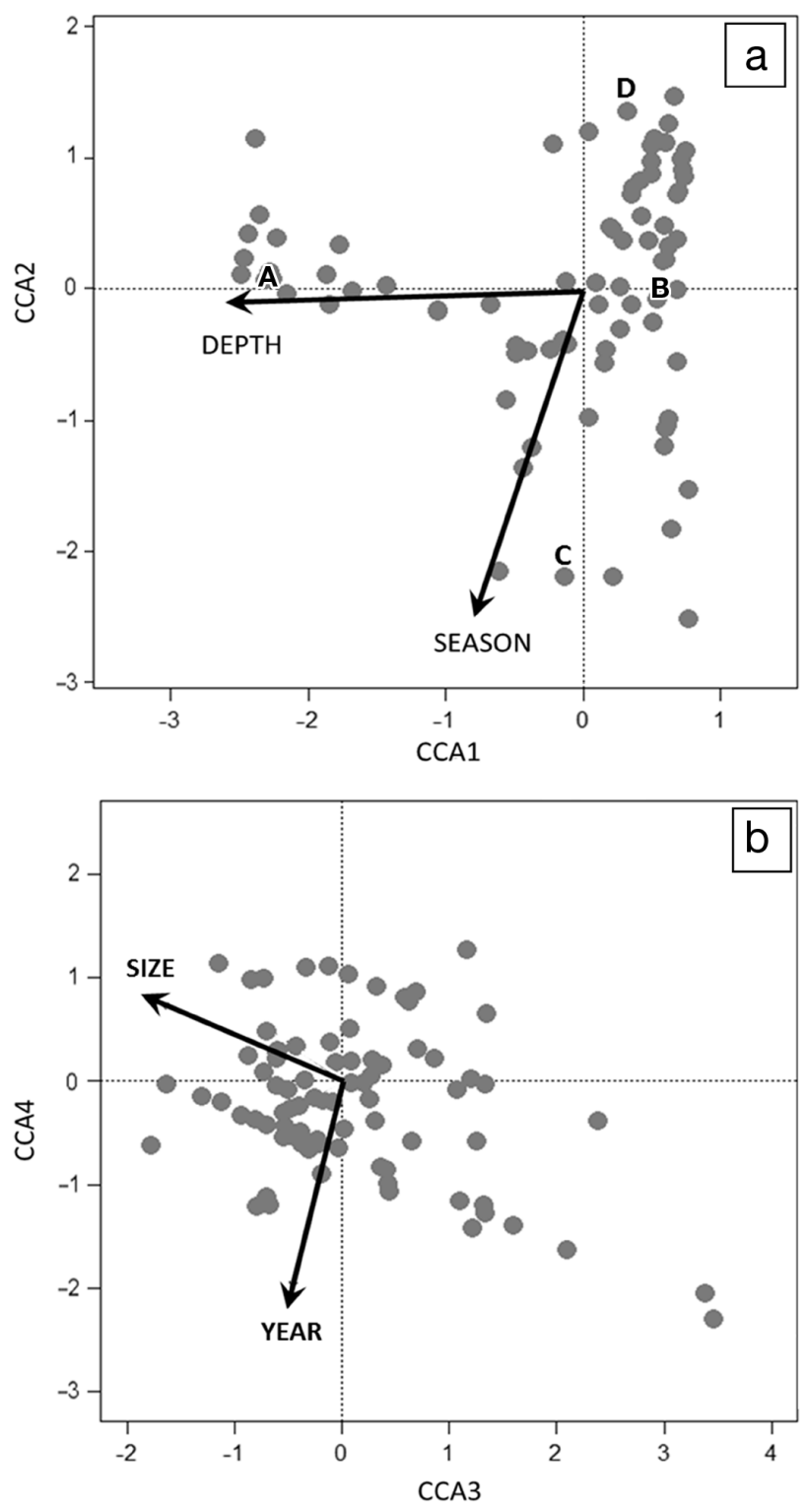

Fig. 4. Canonical correspondence analysis biplots. Data points represent individual prey groups. Arrows represent explanatory variables for (a) depth and season and (b) size and year, and their relationship to the distribution of prey groups. A correlation between an explanatory variable and a biplot axis is indicated by a small angle between them. A: squat lobster Munida simplex; B: nimble spray crab Percnon gibbesi; C: belted cardinalfish Apogon townsendi; D: pearl blenny Entomacrodus nigricans

reported by Morris \& Akins (2009) in the Bahamas, where lionfish consumed more than 4 times the teleosts $\left(78.0 \% V^{\mathbf{1}}\right)$ relative to crustaceans $\left(14.4 \% V^{\underline{1}}\right)$.

\footnotetext{
${ }^{\mathbf{1}}$ As the average density of prey items was approximately $1 \mathrm{~g}$ $\mathrm{cm}^{-3}$, we assume the percent composition by volume $(\% \mathrm{~V})$ and $\% M$ are equivalent, thus allowing the comparison of $\% M$ from our study with $\% V$ in others
}

Table 4. Comparison of the 3 common diet metrics - percent composition by number $(\% N)$, percent frequency of occurrence $(\% F)$, and percent composition by mass $(\% M)-$ of lionfish (Pterois volitans and P. miles) between different regions throughout the northwest Atlantic Ocean

\begin{tabular}{|c|c|c|c|c|}
\hline & & $\% N$ & $\% M$ & $\% F$ \\
\hline \multirow{2}{*}{ Bermuda } & Teleost & 55.5 & 73.4 & 51.9 \\
\hline & Crustacean & 43.0 & 23.4 & 33.8 \\
\hline \multirow{2}{*}{ Bahamas } & Teleost & 71.2 & $78.0^{\mathrm{a}}$ & 61.6 \\
\hline & Crustacean & 28.5 & $14.4^{\mathrm{a}}$ & 24.7 \\
\hline \multirow[t]{2}{*}{ Mexican-Caribbean } & Teleost & 74.4 & & \\
\hline & Crustacean & 25.6 & & \\
\hline \multirow{2}{*}{ Southeast USA ${ }^{\mathrm{b}}$} & Teleost & 84.1 & $86.5^{\mathrm{a}}$ & \\
\hline & Crustacean & 13.9 & $11.5^{\mathrm{a}}$ & \\
\hline \multicolumn{5}{|c|}{$\begin{array}{l}\text { a This study used } \% V \text { in lieu of } \% M \\
\text { bData from Muñoz et al. (2011) }\end{array}$} \\
\hline
\end{tabular}

Muñoz et al. (2011) reported an even greater contribution of teleosts to the diet of lionfish along the US coast (86.5\% $M$ teleosts vs. $11.5 \% M$ crustaceans). Nonetheless, crustaceans still make a more substantial contribution to the diet of lionfish in Bermuda than elsewhere. In total, 22 families of teleosts (44 species) and 14 families of crustaceans (17 species) were found in the stomach contents of lionfish in Bermuda. This substantial contribution of both teleosts and crustaceans, with a broad range of families and species in each category, supports the prevailing view that lionfish are generalist and opportunist predators (Muñoz et al. 2011, Layman \& Allgeier 2012, Côté et al. 2013).

The contribution of key prey items was fairly consistent across the indices of importance, at the species and family level, and when considering trophic guild (Table 3). Despite some limited variation in the order by which items were ranked, 9 species and 8 families were consistently represented in each index, and the 2 top-ranked species and their families were consistent across all metrics (Table 3). Similarly, invertivores (60.4\%IRI) and detrivores (27.3\% IRI) were the 2 top-ranked trophic guilds consumed by lionfish, while herbivores ranked third (6.9\%IRI). The consistency of the rankings among the indices indicates that the red night shrimp Cinetorhynchus rigens is the most important prey species for lionfish in Bermuda (Table 3). This conclusion is reinforced by its substantial contribution in number, mass, and frequency and its ubiquitous presence in lionfish stomachs throughout the year and at all depths. Even at the family level, Rhynchocinetidae (to which the red night shrimp belongs) makes the greatest contribution to the lionfish diet (Table 3 ). This is the first 
study to show a crustacean species, and its related family, playing such a substantial and extensive role in the diet of lionfish.

The red night shrimp makes the biggest contribution to the diet of lionfish in Bermuda for any single prey item, approximately 2 to 3 times greater than the second item by all 3 diet metrics (Table 2). This is notable considering their minimal contribution $\left(0.5 \% N\right.$ and $\left.1.0 \% V^{\mathbf{1}}\right)$ recorded in the Bahamas (Morris \& Akins 2009) and their absence from lionfish diet in the Gulf of Mexico and along the southeastern coast of the USA (Muñoz et al. 2011, Dahl \& Patterson 2014). Because lionfish and red night shrimp both inhabit deep crevices and caves during the day and actively feed at night (Burkenroad 1939, Fishelson 1975), this spatial and temporal habitat overlap likely increases the frequency of encounters between the 2 species, potentially contributing to the high consumption of red night shrimp. Alternatively, in the absence of data that describe the populations of red night shrimp in Bermuda or elsewhere, this could potentially reflect their abundance in Bermuda, which lionfish exploit opportunistically. Considering their significant presence in the diet of lionfish in Bermuda, if these shrimp have not previously been the target of native predators, the possibility exists that lionfish may exert an unsustainable pressure on their population. On the other hand, if red night shrimp are consumed by native predators, there could be consequences for the other species that feed upon them, as they would now be competing with invasive lionfish for those resources. Given the rate at which red night shrimp are being consumed by lionfish in Bermuda, a further examination of their biology and ecology may be warranted to identify the impact lionfish may have on their population and any subsequent effects on Bermuda's coral reef ecosystem.

As in previous studies (Morris \& Akins 2009, Muñoz et al. 2011, Dahl \& Patterson 2014), our work shows that lionfish feed upon juveniles and smallbodied adults of many species. Teleost prey items are not just small, but often shallow-bodied (e.g. wrasse and gobies), a trait shown by Green \& Côté (2014) to be correlated with an increased vulnerability to predation. It is harder to describe the characteristic traits of crustacean prey in a similar manner. For example, the nimble spray crab Percnon gibbesi are flat and wide-bodied, with a row of spines on each walking leg and, in general, do not present themselves as easily consumable prey. In addition, these shore crabs are found along rocky shorelines below the mean low-water level (Sterrer 1986) and often forage in water $<30 \mathrm{~cm}$ deep (C. Eddy pers. obs.). Despite these potentially unappealing morphological traits and their shallow habitat, nimble spray crabs ranked as the $7^{\text {th }}$ (IOP) and $8^{\text {th }}(\%$ IRI) (Table 3) relatively important prey item because they made a large contribution to diet for lionfish captured at one specific site ( 3 to $7 \mathrm{~m}$ deep) where a shallow rocky coastline is immediately adjacent to high-relief reefs.

Considering the contrasting morphological shapes and behaviors of teleost and crustacean prey, it seems other factors may be contributing to the increased consumption of the latter. A greater abundance of crustacean prey relative to teleosts at certain times of the day (e.g. crepuscular periods) or in certain locations (e.g. deep crevices and caves) is the most parsimonious answer. However, prey naïveté may also be contributing to the heavy predation on some of these otherwise risk-averse crustaceans. Cryptic and shy, red night shrimp shelter in recesses and amongst the spines of the long-spined sea urchin Diadema antillarum (Humann \& DeLoach 1992, Hernández 2008). Similarly, the nimble spray crab, which is sometimes known as the urchin crab, is often found to associate with this same species (Hayes et al. 1998). To naïve prey with these sheltering instincts, the elongate dorsal and pectoral fins of lionfish may appear to be a similarly benign structural refuge, perhaps increasing their exposure to predation. Alternatively, as lionfish are visual predators (Fishelson 1997), the movement of some crustaceans (e.g. hurried motions, frequent rapid changes in direction) may stimulate an instinctive predatory response from the lionfish that increases their vulnerability.

Lionfish in Bermuda consume more than 60 different prey species that play important roles in coral reef communities. While the Atlantic creolefish Paranthias furcifer is the only economically valuable species among the top 10 prey items for lionfish in Bermuda (Tables $2 \& 3$ ), other prey items play vital ecological roles, such as juvenile bluehead wrasse Thalassoma bifasciatum, which provides a beneficial service to reef fish by cleaning them of dead skin and ectoparasites (Feddern 1965). Although herbivorous teleosts (e.g. juvenile scarids and acanthurids), which help maintain coral health and facilitate coral recruitment by grazing upon algae, seem to be consumed infrequently in Bermuda (at least at present), the possibility exists that lionfish diet may change from year to year as prey availability changes (Muñoz et al. 2011). While the ecological roles of some species, in particular the crustaceans, are relatively unknown, the taxa consumed by lionfish in Bermuda include a widely diverse assortment of scavengers and detriti- 
vores, zooplanktivores, planktivores, herbivores, and piscivores. The prey data in the current study show that most trophodynamic guilds are represented in the lionfish diet, reinforcing the concern that these invasive teleosts may have the potential to disrupt normal ecosystem function. Furthermore, many lionfish prey species could be essential prey for higherlevel predators, including some of economic importance (e.g. snappers) (Muñoz-Escobar \& Gil-Agudelo 2012).

Lionfish diet in Bermuda is affected by both depth and season, presumably due to spatial and temporal variation in community structure and, therefore, prey availability driven by those environmental factors (MacNeil \& Connolly 2015). The first axis of the CCA bi-plot is correlated with depth, which is likely due to variations in habitat preferences and depth distributions of various prey species (Fig. 4a). For example, certain prey species (e.g. squat lobster) are only encountered at $60 \mathrm{~m}$, while other species (e.g. nimble spray crab and seaweed blenny Parablennius marmoreus) were only found within stomachs of lionfish captured in shallow waters (i.e. $<30 \mathrm{~m}$ ). Similarly, Atlantic creolefish are abundant at $60 \mathrm{~m}$ and their juveniles are one of the most common prey items for lionfish captured at these depths. For this reason, it is important to consider that this species may be significantly impacted by lionfish, which occur in very high densities at these depths in Bermuda (Department of Environment and Natural Resources unpubl. data). The second axis of the CCA bi-plot (Fig. 4a) corresponds with season, suggesting that there are also seasonal trends in lionfish diet. For example, certain prey items (e.g. pearl blenny Entomacrodus nigricans and belted cardinalfish Apogon townsendi) were only found in the stomachs of lionfish during specific times of the year (e.g. mid-winter and midautumn, respectively), indicating that there may be seasonal changes in prey availability that could not be detected adequately with the visual prey surveys. This could also be indicative of a seasonal movement between different habitats, for which we already have anecdotal evidence, but which would have to be explored directly in the future.

Another factor that influences diet is the size of the lionfish. As the third CCA axis was significantly correlated with lionfish size (Fig. $4 \mathrm{~b}$ ) and the mean values of all 3 diet metrics (i.e. $\% N, \% M, \% F$ ) showed an increase in the contribution of teleosts among larger lionfish (Fig. 3), it appears lionfish undergo an ontogenetic diet shift from crustaceans to teleosts. This pattern has been previously recognized in lionfish from the Bahamas (Morris \& Akins 2009) and could be explained by a number of factors. Since lionfish are a gape-limited predator (Côté et al. 2013), the diet shift may reflect an increase in the maximum size of potential prey as lionfish gape increases, and a limit to the minimum size of prey as small items may escape through the gaps between gill rakers (Graham et al. 2007). Further, as larger lionfish have greater gross energetic requirements, a switch to teleosts may provide greater mass-specific nutritional benefits compared to crustaceans. It should be noted, however, that the current study found a large $308 \mathrm{~mm}$ (TL) lionfish with 27 squat lobsters in its stomach and another larger $442 \mathrm{~mm}$ (TL) individual with a single red night shrimp in its stomach. Thus, while larger lionfish may consume a greater proportion of teleosts, it does not preclude them from opportunistically feeding on crustaceans. We propose that the ontogenetic diet shift in lionfish reflects their capacity to exploit an increasing variety of resources, effectively expanding their dietary niche and spreading their impact across a broad range of species, both large and small.

Given the influence of depth on the diet of lionfish, it is important to note that stomachs of lionfish caught in deep water $(>30 \mathrm{~m}$ ) were more than twice as likely to be empty $(61.6 \%)$ compared to those captured in shallow water $(<30 \mathrm{~m} ; 28.1 \%)$. As our surveys suggest potential prey items are abundant at both shallow and deep sites, the higher proportion of empty stomachs from individuals captured in deeper waters may have resulted from decompression-related barotrauma, regurgitation, and stomach eversion during the ascent following specimen collection (DeMartini et al. 1996). We thus suggest that future work aiming to develop a more complete understanding of lionfish diet and their potential impacts on the reef ecosystem should include methods that prevent the loss of stomach contents from lionfish captured in deep water.

Considering the broad diversity of prey items for lionfish in Bermuda and the large quantity of welldigested items that could not be identified in the stomachs, it is possible that some prey species were not recognized and accounted for, and that the diet of lionfish in Bermuda is even broader than characterized here. As such, the economically important species previously documented in the diet of lionfish elsewhere (e.g. juvenile groupers and snappers in the Bahamas; Morris \& Akins 2009) may not be exempt from predation in Bermuda even though they went undetected in the current study. It is also possible that we simply did not sample lionfish from areas where juveniles of these species are common. The current study focused along the outer perimeter of 
Bermuda's reef platform as there were very limited reports of lionfish in the lagoon and inshore waters (Fig. 1) compared to observations of higher densities along the outer and deep reefs. However, in Bermuda, juveniles of many teleost families recruit to inshore and lagoonal patch reefs far more frequently than to rim and terrace reefs (Smith et al. 2013). For this reason, additional efforts should be made to search for and collect lionfish from inshore and lagoonal habitats to more completely investigate their presence in these critical nursery areas and their potential impact on the biodiversity of both ecologically and economically important species. Taken together, these data suggest that if the lionfish population in Bermuda continues to expand, there will likely be no spatial or temporal refuge for potential lionfish prey, which may eventually include any juvenile or small-bodied fishes or crustaceans. This, in turn, suggests that the impacts of invasive lionfish may eventually be felt across a broad range of species and habitats, as has indeed been suggested by other studies (see for example Muñoz et al. 2011, Côté et al. 2013).

\section{CONCLUSIONS}

The extent to which invasive lionfish may impact Bermuda's coral reef ecosystem is still relatively unknown. While evidence suggests that, to date, their population has not experienced the explosive growth seen in other regions, there is concern that this may be coming soon, with wide-scale impacts on biodiversity, community structure, and ecosystem function. This study provides information to help resource managers evaluate which prey species may be most vulnerable to lionfish predation and therefore the species and habitats that may suffer the greatest impact. Our work suggests that small crustacean species will experience considerable impacts, but the consequences of the intensive selection for these species remains unknown. Overall, the diet of lionfish seems to be spread across a broad range of species, perhaps minimizing their current impact on individual species. As the lionfish population grows in Bermuda, this may change. Although lionfish are consuming some ecologically important teleosts, herbivorous species (e.g. parrotfish) appear to be targeted less often, suggesting a phase shift to an algaedominated community may not be an immediate concern. However, a commercially important serranid (Atlantic creolefish) is highly-targeted in its juvenile deep reef habitat (i.e. $60 \mathrm{~m}$ ). If this remains the case, there may be less of a direct impact upon the health of the coral reef itself, although the negative influence upon other teleosts, including top predators, may increase. Of course, if the progress of the lionfish invasion in Bermuda accelerates, these scenarios may change dramatically.

Acknowledgements. Funding was provided by the National Science Foundation (DGE-1144241); the UK's Department for Environment, Food, and Rural Affairs (DEFRA) (DPLUS001); and the University of Massachusetts Dartmouth. The Bermuda Zoological Society helped administer and manage the DEFRA grant. Logistic support was provided by the National Oceanic and Atmospheric Administration (NOAA), Bermuda's Department of Environment and Natural Resources (DENR), and the Ocean Support Foundation. The authors thank the NOAA reviewers for their valuable comments as we revised the manuscript. The authors also thank Mandy Shailer from the DENR for her assistance with GIS and mapping. The authors are especially grateful to all lionfish hunters in the Bermuda Lionfish Culling Program, and those commercial fishermen who provided lionfish for this study. In particular, we thank Weldon Carl Wade, the Lionfish Mafia (William Gillett, Jon Pedro, Adrienne Smatt, Andrew Conyers, and Andrew de Melo), Adrian Kawaley-Lathan, Michael Sinclair, Tommy Sinclair, Joshua Hill, Joshua Stephens, and (last, but unquestionably not least) Mark Outerbridge, Chris Cabral, and Alex Davidson for their help.

\section{LITERATURE CITED}

Albins MA, Hixon MA (2008) Invasive Indo-Pacific lionfish Pterois volitans reduce recruitment of Atlantic coral-reef fishes. Mar Ecol Prog Ser 367:233-238

Albins MA, Hixon MA (2013) Worst case scenario: potential long-term effects of invasive predatory lionfish (Pterois volitans) on Atlantic and Caribbean coral-reef communities. Environ Biol Fishes 96:1151-1157

Albins MA, Lyons PJ (2012) Invasive red lionfish Pterois volitans blow directed jets of water at prey fish. Mar Ecol Prog Ser 448:1-5

Bethea DM, Carlson JK, Hollensead LD, Papastamatiou YP, Graham BS (2011) A comparison of the foraging ecology and bioenergetics of the early life-stages of two sympatric hammerhead sharks. Bull Mar Sci 87:873-889

Bizzarro JJ, Robinson HJ, Rinewalt CS, Ebert DA (2007) Comparative feeding ecology of four sympatric skate species off central California. Environ Biol Fishes 80: 197-220

Bohlke JE, Chaplin CC (1993) Fishes of the Bahamas and adjacent tropical waters. University of Texas Press, Austin, TX

Burkenroad MD (1939) Some remarks upon non-peneid Crustacea Decapoda. J Nat Hist 3:310-318

Cartes JE (1993) Diets of two deep-sea decapods: Nematocarcinus exilis (Caridea: Nematocarcinidae) and Munida tenuimana (Anomura: Galatheidae) on the western Mediterranean slope. Ophelia 37:213-229

Cervigón FR (1993) Los Peces Marinos de Venezuela. Fundación Científica Los Roques, Caracas

Chande AI, Mgaya YD (2005) Food habits of the blue 
swimming crab Portunus pelagicus along the coast of Dar es Salaam, Tanzania. J Mar Sci 3:37-42

Corredor L (1978) Notes on the behavior and ecology of the new fish cleaner shrimp Brachycarpus biunguiculatus (Lucas) (Decapoda Natantia, Palaemonidae). Crustaceana 35:35-40

Cortés E (1997) A critical review of methods of studying fish feeding based on analysis of stomach contents: application to elasmobranch fishes. Can J Fish Aquat Sci 54: 726-738

Côté IM, Green SJ, Morris JA Jr, Akins JL, Steinke D (2013) Diet richness of invasive Indo-Pacific lionfish revealed by DNA barcoding. Mar Ecol Prog Ser 472:249-256

Cure K, Benkwitt CE, Kindinger TL, Pickering EA, Pusack TJ, Mcllwain JL, Hixon MA (2012) Comparative behavior of red lionfish Pterois volitans on native Pacific versus invaded Atlantic coral reefs. Mar Ecol Prog Ser 467:181-192

Dahl KA, Patterson WF (2014) Habitat-specific density and diet of rapidly expanding invasive red lionfish, Pterois volitans, populations in the northern Gulf of Mexico. PLoS ONE 9:e105852

DeMartini EE, Parrish FA, Ellis DM (1996) Barotraumaassociated regurgitation of food: implications for diet studies of Hawaiian pink snapper, Pristipomoides filamentosus (family Lutjanidae). Fish Bull 94:250-256

Edwards MA, Frazer TK, Jacoby CA (2014) Age and growth of invasive lionfish (Pterois spp.) in the Caribbean Sea, with implications for management. Bull Mar Sci 90: 953-966

Feddern HA (1965) The spawning, growth, and general behavior of the bluehead wrasse, Thalassoma bifasciatum (Pisces: Labridae). Bull Mar Sci 15:896-941

- Ferreira CEL, Luiz OJ, Floeter SR, Lucena MB, Barbosa MC, Rocha CR, Rocha LA (2015) First record of invasive lionfish (Pterois volitans) for the Brazilian coast. PLOS ONE 10:e0123002

Fishelson L (1975) Ethology and reproduction of pteroid fishes found in the Gulf of Aqaba (Red Sea) especially Dendrochirus brachypterus (Cuvier) (Pteroidae, Teleostei). Pubbl Stn Zool Napoli 39:635-656

- Fishelson L (1997) Experiments and observations on food consumption, growth and starvation in Dendrochirus brachypterus and Pterois volitans (Pteroinae, Scorpaenidae). Environ Biol Fishes 50:391-403

Frazer TK, Jacoby CA, Edwards MA, Barry SC, Manfrino CM (2012) Coping with the lionfish invasion: Can targeted removals yield beneficial effects? Rev Fish Sci 20: 185-191

Frick MG, Williams KL, Bolten AB, Bjorndal KA, Martins HR (2004) Diet and fecundity of Columbus crabs, Planes minutus, associated with oceanic-stage loggerhead sea turtles, Caretta caretta, and inanimate flotsam. J Crustac Biol 24:350-355

Gardner PG, Frazer TK, Jacoby CA, Yanong RP (2015) Reproductive biology of invasive lionfish (Pterois spp.). Front Mar Sci 2:1-10

Graham BS, Grubbs D, Holland K, Popp BN (2007) A rapid ontogenetic shift in the diet of juvenile yellowfin tuna from Hawaii. Mar Biol 150:647-658

> Green SJ, Côté IM (2009) Record densities of Indo-Pacific lionfish on Bahamian coral reefs. Coral Reefs 28:107

Green SJ, Côté IM (2014) Trait-based diet selection: prey behaviour and morphology predict vulnerability to predation in reef fish communities. J Anim Ecol 83: 1451-1460
Green SJ, Akins JLM, Maljković A, Côté IM (2012) Invasive lionfish drive Atlantic coral reef fish declines. PLOS ONE 7:e32596

Hayes FE, Joseph VL, Gurley HS, Wong BYY (1998) Selection by two decapod crabs (Percnon gibbesi and Stenorhynchus seticornis) associating with an urchin (Diadema antillarum) at Tobago, West Indies. Bull Mar Sci 63:241-247

Hernández MS (2008) Relaciones interespecíficas entre Diadema antillarum (Echinodermata: Diadematidae) y otros invertebrados a lo largo del ciclo lunar. An Univ Etol 2:62-70

> Hughes RN, Elner RW (1989) Foraging behaviour of a tropical crab: Calappa ocellata (Holthuis) feeding upon the mussel Brachidontes domingensis (Lamarck). J Exp Mar Biol Ecol 133:93-101

Humann P, DeLoach N (1992) Reef creature identification: Florida, Caribbean, Bahamas. New World Publications, Jacksonville, FL

- Hyslop EJ (1980) Stomach contents analysis - a review of methods and their application. J Fish Biol 17:411-429

Iversen ES, Jory DE, Bannerot SP (1986) Predation on queen conchs, Strombus gigas, in the Bahamas. Bull Mar Sci 39:61-75

Jackson JBC, Donovan MK, Cramer KL, Lam VV (2014) Status and trends of Caribbean coral reefs: 1970-2012. Global Coral Reef Monitoring Network, IUCN, Gland

Jongman RH, ter Braak CJF, Tongeren OFR (eds) (1987) Data analysis in community and landscape ecology. Cambridge University Press, Cambridge

Kendall JJ (1990) Further evidence of cooperative foraging by the turkeyfish Pterois miles in the Gulf of Aqaba, Red Sea, with comments on safety and first aid. Proc Am Acad Underwater Sci Scientific Diving Symp 10: 209-223

Lavalli KL, Spanier E, Grasso F (2007) Behavior and sensory biology of slipper lobsters. In: Lavalli KL, Spanier E (eds) The biology and fisheries of the slipper lobster. CRC Press, Boca Raton, FL, p 133-181

> Layman CA, Allgeier JE (2012) Characterizing trophic ecology of generalist consumers: a case study of the invasive lionfish in The Bahamas. Mar Ecol Prog Ser 448:131-141

> Lesser MP, Slattery M (2011) Phase shift to algal dominated communities at mesophotic depths associated with lionfish (Pterois volitans) invasion on a Bahamian coral reef. Biol Invasions 13:1855-1868

Luiz OJ, Floeter SR, Rocha LA, Ferreira CEL (2013) Perspectives for the lionfish invasion in the South Atlantic: Are Brazilian reefs protected by the currents? Mar Ecol Prog Ser 485:1-7

Mack RN, Simberloff D, Lonsdale MW, Evans H, Clout M, Bazzaz FA (2000) Biotic invasions: causes, epidemiology, global consequences, and control. Ecol Appl 10:689-710

MacNeil MA, Connolly SR (2015) Multi-scale patterns and processes in reef fish abundance. In: Camilo M (ed) Ecology of fishes on coral reefs. Cambridge University Press, Cambridge, p 116-124

McEachran JD (2009) Fishes (Vertebrata: Pisces) of the Gulf of Mexico. In: Felder DL, Camp DK (eds) Gulf of Mexico origins, waters, and biota, Vol 1: biodiversity. Texas A\&M University Press, College Station, TX, p 1223-1316

Molnar JL, Gamboa RL, Revenga C, Spalding MD (2008) Assessing the global threat of invasive species to marine biodiversity. Front Ecol Environ 6:485-492

Morris JA (2009) The biology and ecology of the invasive 
Indo-Pacific lionfish. PhD dissertation, North Carolina State University, Raleigh, NC

Morris JA, Akins JL (2009) Feeding ecology of invasive lionfish (Pterois volitans) in the Bahamian Archipelago. Environ Biol Fishes 86:389-398

Morris JA, Whitfield PE (2009) Biology, ecology, control and management of the invasive Indo-Pacific lionfish: an updated integrated assessment. NOAA Tech Memo NOS NCCOS 99. National Oceanic and Atmospheric Administration, Washington, DC

Muñoz RC, Currin CA, Whitfield PE (2011) Diet of invasive lionfish on hard bottom reefs of the Southeast USA: insights from stomach contents and stable isotopes. Mar Ecol Prog Ser 432:181-193

Muñoz-Escobar L, Gil-Agudelo DL (2012) Composicion dietaria del pez leon, Pterois volitans (Pisces: Scorpaenidae), en Santa Marta y el Parque Nacional Natural Tayrona. Bol Invest Mar Cost 41:471-477

Natrajan AV, Jhingran AG (1962) Index of preponderance: a method of grading the food elements in the stomach analysis of fishes. Ind J Fish 8:54-59

Pinkas LM, Oliphant S, Iverson ILK (1971) Food habits of albacore, bluefin tuna and bonito in Californian waters. Calif Fish Game 152:1-105

Puccio V, Relini M, Azzurro E, Relini LO (2006) Feeding habits of Percnon gibbesi (H. Milne Edwards, 1853) in the Sicily Strait. Hydrobiologia 557:79-84

Randall JE (1967) Food habits of reef fishes of the West Indies. Stud Trop Oceanogr 5:665-847

Robertson DR (1981) The social and mating systems of two labrid fishes, Halichoeres maculipinna and H. garnoti, off the Caribbean coast of Panama. Mar Biol 64:327-340

Romero MC, Lovrich GA, Tapella F, Thatje S (2004) Feeding ecology of the crab Munida subrugosa (Decapoda: Anomura: Galatheidae) in the Beagle Channel, Argentina. J Mar Biol Assoc UK 84:359-365

Ryan EP (1956) Observations on the life histories and the distribution of the Xanthidae (mud crabs) of Chesapeake Bay. Am Midl Nat 56:138-162

Sakai AK, Allendorf FW, Holt JS, Lodge DM and others (2001) The population biology of invasive species. Annu Rev Ecol Syst 32:305-332

Samson SA, Yokota M, Strüssmann CA, Watanabe S (2007) Natural diet of grapsoid crab Plagusia dentipes de Haan (Decapoda: Brachyura: Plagusiidae) in Tateyama Bay, Japan. Fish Sci 73:171-177

Schofield PJ (2010) Update on geographic spread of invasive lionfishes (Pterois volitans [Linnaeus, 1758] and P. miles

Editorial responsibility: Mark Hixon (Guest Editor),

Honolulu, Hawaii, USA
[Bennett, 1828]) in the Western North Atlantic Ocean, Caribbean Sea and Gulf of Mexico. Aquat Invasions 5: S117-S122

Schultz ET, Cowen RK (1994) Recruitment of coral reef fishes to Bermuda: local retention or long-distance transport? Mar Ecol Prog Ser 109:15-28

Smith SR, de Putron S, Murdoch TJ, Pitt JM, Nagelkerken I (2013) Biology and ecology of corals and fishes on the Bermuda Platform. In: Sheppard CRD (ed) Coral Reefs of the United Kingdom overseas territories. Springer, Dordrecht, p 135-151

Sterrer W (1986) Marine flora and fauna of Bermuda: a systematic guide to the identification of marine organisms. Island Press, Bermuda

Sterrer W (1992) Bermuda's marine life. Bermuda Zoological Society. Island Press, Bermuda

ter Braak CJF (1986) Canonical correspondence analysis: a new eigenvector technique for multivariate direct gradient analysis. Ecology 67:1167-1179

$>$ ter Braak CJF (1987) The analysis of vegetation-environment relationships by canonical correspondence analysis. Vegetatio 69:69-77

Valdez-Moreno M, Quintal-Lizama C, Gómez-Lozano R, del Carmen García-Rivas M (2012) Monitoring an alien invasion: DNA barcoding and the identification of lionfish and their prey on coral reefs of the Mexican Caribbean. PLOS ONE 7:e36636

Vitousek PM, D'antonio CM, Loope LL, Rejmanek M, Westbrooks R (1997) Introduced species: a significant component of human-caused global change. NZ J Ecol 21:1-16

Whiteman EA, Côté IM, Reynolds JD (2007) Ecological differences between hamlet (Hypoplectrus: Serranidae) colour morphs: between-morph variation in diet. J Fish Biol 71:235-244

> Whitfield PE, Gardner T, Vives SP, Gilligan MR, Courtenay WR Jr, Ray GC, Hare JA (2002) Biological invasion of the Indo-Pacific lionfish Pterois volitans along the Atlantic coast of North America. Mar Ecol Prog Ser 235: 289-297

Whitfield PE, Hare JA, David AW, Harter SL, Muñoz RC, Addison CM (2007) Abundance estimates of the IndoPacific lionfish Pterois volitans/miles complex in the Western North Atlantic. Biol Invasions 9:53-64

Zar JH (1999) Biostatistical analysis. Prentice Hall, Upper Saddle River, NJ

Z Zhang D, Lin J, Creswell L (1998) Mating behavior and spawning of the banded coral shrimp Stenopus hispidus in the laboratory. J Crustac Biol 18:511-518

Submitted: January 20, 2016; Accepted: July 18, 2016 Proofs received from author(s): September 2, 2016 